\title{
Development of a novel hepatitis $B$ virus encapsidation detection assay by viral nucleocapsid-captured quantitative RT-PCR
}

\author{
Dong-Kyun Ryu ${ }^{1, \dagger}$, Yeji Ahn², Wang-Shick Ryu², and Marc P. Windisch ${ }^{1}$ \\ ${ }^{1}$ Hepatitis Research Laboratory, Institut Pasteur Korea, Seongnam-si, Gyeonggi-do, \\ South-Korea and '2Department of Biochemistry, Yonsei University, Seoul, South-Korea \\ ${ }^{\dagger}$ Present address: Celltrion Inc., Academy-ro 51, Yeonsu-gu, Incheon, 406-840 South-Korea.
}

BioTechniques 59:287-293 (November 2015) doi 10.2144/000114354

Keywords: hepatitis B virus; pregenomic RNA; encapsidation; antiviral assay

After encapsidation, where pregenomic RNA (pgRNA) is packaged into viral nucleocapsids, hepatitis $B$ virus (HBV) uses the pgRNA as a template to replicate its DNA genome by reverse transcription. To date, there are only two encapsidation detection methods for evaluating the amount of pgRNA packaged into nucleocapsids: (i) the RNase protection assay and (ii) the native agarose gel electrophoresis assay. However, these methods are complex and laborious because they require multiple pgRNA purification steps followed by detection via an isotope-labeled probe. Moreover, both assays are unsuitable for evaluating a large number of antiviral agents in a dose-dependent manner. To overcome these limitations, we devised a novel HBV encapsidation assay in a 96-well plate format using nucleocapsid capture plates coated with an anti-HBV core (HBc) antibody, usually employed in enzyme-linked immunosorbent assays, to immobilize viral nucleocapsids. Viral pgRNA is then detected by quantitative RT-PCR (RT-qPCR). This strategy allows fast, convenient, and quantitative analysis of multiple viral RNA samples to evaluate encapsidation inhibitors. Furthermore, our protocol is potentially suitable for high-throughput screening (HTS) of compounds targeting HBV pgRNA encapsidation.

More than 350 million people worldwide are chronically infected with the hepatitis B virus (HBV) and are at risk of developing life-threatening liver diseases such as liver cirrhosis and hepatocellular carcinoma (1). Despite the availability of several medications and vaccines, chronically infected patients cannot be cured and require long-term treatment with antivirals, which could eventually lead to drug resistance (2). HBV is a small enveloped virus belonging to the family Hepadnaviridae. Because the viral relaxed, circular $(\mathrm{RC})$ and partially double-stranded DNA (dsDNA) genome of $\sim 3 \mathrm{~kb}$ is replicated by reverse transcription through an RNA intermediate, $\mathrm{HBV}$ is classified as a para-retrovirus. This hepatotropic virus exclusively replicates in the cytoplasm of hepatocytes, where pregenomic RNA (pgRNA) is selectively encapsidated together with the viral polymerase (Pol) into HBV nucleocapsids (3-5). Encapsidation of pgRNA requires the recognition and binding of Pol to a stem-loop structure called epsilon $(\varepsilon)$ located at the $5^{\prime}$ end of the pgRNA (3). Interaction of the $\varepsilon$-Pol ribonucleoprotein complex inhibits viral translation and triggers capsid assembly by specific incorporation of both pgRNA and Pol into viral nucleocapsids where reverse transcription takes place to form the mature RC DNA genome (3-5).

To date, there are only two methods for monitoring viral pgRNA encapsidation into nucleocapsids: (i) the RNase protection assay and (ii) the native agarose gel electrophoresis assay (6-11). Both assays require complex, laborious, and time-consuming experimental steps to purify and analyze viral RNA containing nucleocapsids. Briefly, the encapsidated pgRNA is purified from cytoplasmic nucleocapsids using either polyethylene glycol (PEG) or anti-HBV core (HBc) antibody. In order to purify viral nucleocapsids using PEG, HBV replicating cells are lysed, treated with nuclease to remove cellular DNA and RNA, and nucleocapsids are precipitated by PEG. Alternatively, to purify nucleocapsids using antibodies, the HBV producing cells are lysed, incubated

\section{METHOD SUMMARY}

Here we present a novel encapsidation assay for hepatitis B virus (HBV), employing quantitative RT-PCR (RT-qPCR) of viral pregenomic RNA (pgRNA) isolated from nucleocapsids captured by an anti-HBV core (HBc) antibody. This technique, which supports a 96-well plate format, allows the evaluation of a large number of antiviral compounds in a dose-dependent manner in parallel and is potentially compatible with high-throughput screening. 
with anti-HBc antibody, and viral nucleocapsids are enriched by protein $\mathrm{G}$. Afterwards, the viral pgRNA inside nucleocapsids prepared by either the PEG or anti-HBc antibody methods is extracted via acid guanidinium thiocyanate-phenol-chloroform extraction (12).

For encapsidation analysis by RNase protection assay, the extracted pgRNA is hybridized to an HBV-specific in vitrotranscribed isotope-labeled RNA probe. Following digestion with RNase $\mathrm{A} / \mathrm{T} 1$, the protected pgRNA is precipitated, subjected to acrylamide gel electrophoresis, and analyzed by phosphoimaging. For encapsidation analysis employing native agarose gel electrophoresis, the collected nucleocapsids are subjected to electrophoresis and blotted to a membrane, followed by lysis of transferred nucleocapsids to expose the packaged pgRNA. In order to detect the blotted pgRNA on the membrane, in vitrotranscribed isotope-labeled probes are used for hybridization. Because of these multiple experimental steps, both encapsidation detection protocols are prone to variation and restrict the evaluation of a large number of samples in a dose-dependent manner due to multiple labor-intensive experimental steps and gel electrophoresis-based read-out. Furthermore, by using radioisotopes, occupational radiation exposure risks as well as costs for appropriate facilities and decay storage are of concern.

These limitations prompted us to develop a more convenient assay in 96-well plates, supporting the analysis of a large number of samples in a dose-dependent manner to investigate HBV pgRNA encapsidation inhibition. Here, we report the development of a novel nucleocapsid-captured quantitative RT-PCR (NCC RT-qPCR) assay. Our strategy is based on the utilization of capture plates, usually used for enzyme-linked immunosorbent assay (ELISA), to immobilize HBV nucleocapsids, followed by the detection of viral pgRNA via RT-qPCR, which requires significantly fewer experimental steps than previously reported methodologies and thus increases the number of inhibitors that can be tested simultaneously.

\section{Materials and methods}

Cell culture and compound treatment HepG2.2.15 cells, stably replicating and secreting HBV, were cultured in Dulbecco's modified Eagle's medium (DMEM) (LM001-07; Welgene, Daegu, Korea) supplemented with $10 \%$ fetal bovine serum (FBS)
(A15-751; PAA, Cölbe, Germany), 400 g/ mL G418 (Geneticin antibiotic) (Invitrogen, Carlsbad, CA), and penicillin-streptomycin (Invitrogen) at $37^{\circ} \mathrm{C}$ in a $5 \% \mathrm{CO}_{2}$ incubator. DVR-45 (K781-8071; ChemDiv, San Diego, $\mathrm{CA}$ ) and lamivudine (Sigma-Aldrich, St. Louis, MO) were dissolved in DMSO. HepG2.2.15 cells $\left(4 \times 10^{4}\right.$ cells/well) were seeded in a 96-well plate (Greiner, Munich, Germany) and treated with compounds the following day. Every third day, cell culture medium and compounds were replaced. At 9 days posttreatment, viral pgRNA was isolated. HepG2 cells were cultured in DMEM with 10\% FBS at $37^{\circ} \mathrm{C}$ in a $5 \% \mathrm{CO}_{2}$ incubator. The 1.3-mer HBV replicon-transfected cells were treated daily with DVR-45 for 2 days. Three days post-transfection, the cells were harvested and subjected to analysis.

PEG precipitation of HBV cores containing viral pgRNA

Viral pgRNA was isolated via PEG precipitation of HBV nucleocapsids as reported previously (7). HepG2.2.15 cells plated in 12 wells were lysed in $300 \mu \mathrm{L} 1 \%$ NP40 buffer in TNE (10 mM Tris pH 8.0, $100 \mathrm{mM} \mathrm{NaCl}$, $1 \mathrm{mM}$ EDTA) and incubated for $10 \mathrm{~min}$ at $37^{\circ} \mathrm{C}$, followed by centrifugation at 13,000 $\times g$ for 5 min at $4^{\circ} \mathrm{C}$. Clarified samples were incubated with 20 URQ1 RNase-free DNase I (Promega, Madison, WI) and $30 \cup$ micrococcal nuclease (Affymetrix, Santa Clara, $\mathrm{CA}$ ) in the presence of $6 \mathrm{mM} \mathrm{MgCl}_{2}$ and 8 $\mathrm{mM} \mathrm{CaCl}{ }_{2}$ for 30 min at $37^{\circ} \mathrm{C}$ followed by centrifugation at $13,000 \times g$ for $5 \mathrm{~min}$ at $4^{\circ} \mathrm{C}$. Nucleocapsids were precipitated by $100 \mu \mathrm{L}$ 4× PNE buffer (26\% PEG 8000, 1.4 M NaCl, $40 \mathrm{mM}$ EDTA) on ice for $2 \mathrm{~h}$ or overnight. After centrifugation at 13,000 $\times g$ for 15 min at $4^{\circ} \mathrm{C}$, nucleocapsids were resuspended in TNE buffer, and the pgRNA was extracted by TRlzol reagent (Ambion, Austin, TX). Isolated pgRNA was quantified on a NanoVue spectrophotometer (GE Healthcare, Buckinghamshire, UK).

Self-made HBc capture plates and capture of HBV nucleocapsids Polystyrene 96-well plates (Corning, New York) were coated with anti-HBc antibody by incubating with $25 \mu \mathrm{L}$ diluted polyclonal anti-HBc antibody (4 $\mu \mathrm{g} / \mathrm{mL}$ ) (DAKO, Glostrup, Denmark) in antibody coating buffer (ImmunoChemistry Technologies LLC, Bloomington, MN). Assay plates were incubated for $1 \mathrm{~h}$ at room temperature, followed by incubation at $4^{\circ} \mathrm{C}$ overnight. The following day, wells were washed twice with $150 \mu \mathrm{L}$ phosphate-buffered saline
(PBS) including 0.5\% Tween 20 (PBST). Capture plates were blocked with $150 \mu \mathrm{L}$ $1 \%$ BSA in PBST for $1 \mathrm{~h}$ at room temperature, followed by washing as described above. To capture HBV nucleocapsids isolated from HepG2.2.15 cells in 96-well plates, the cells were lysed in $100 \mu \mathrm{L} 1 \%$ NP40 buffer in TNE (10 mM Tris pH 8.0, $100 \mathrm{mM} \mathrm{NaCl}, 1 \mathrm{mM}$ EDTA) for 10 min at $37^{\circ} \mathrm{C}$. Cell lysates were transferred to V-type 96-well plates (Greiner) and subjected to centrifugation at $500 \times g$ for 5 min at $4^{\circ} \mathrm{C}$ to remove nuclei and cell debris. The clarified supernatants were transferred to anti-HBc antibody-coated capture plates, placed on an orbital micro-plate shaker, and incubated for $1 \mathrm{~h}$ at room temperature, followed by a wash step with $150 \mu \mathrm{L}$ PBST. In order to purify pgRNA from nucleocapsids, we employed the CellAmp Direct RNA Prep Kit (TaKaRa Bio, Shiga, Japan) and Proteinase $\mathrm{K}$ (TaKaRa Bio), following the manufacturer's recommendations. Briefly, the assay plate containing the captured nucleocapsids was washed with $125 \mu \mathrm{L}$ CellAmp washing buffer and incubated in $40 \mu \mathrm{L}$ CellAmp processing buffer including Proteinase $\mathrm{K}$ for 5 min at room temperature. The cell lysates were mixed well by pipetting up and down and transferred to a 96-well PCR plate, followed by incubation for $5 \mathrm{~min}$ at $75^{\circ} \mathrm{C}$ in a PCR machine to inactivate Proteinase $\mathrm{K}$. The PCR plate was cooled down on ice, and 10 $\mu \mathrm{L}$ DNase solution, which contains DNase I in CellAmp processing buffer, was added. The plate was incubated for $5 \mathrm{~min}$ at $37^{\circ} \mathrm{C}$ to digest HBV DNA replicative intermediates, followed by incubation at $75^{\circ} \mathrm{C}$ for $5 \mathrm{~min}$ in the PCR machine to inactivate DNase I. The final volume of processed pgRNA per well was $50 \mu \mathrm{L}$.

\section{Quantitative RT-PCR}

To detect HBV pgRNA, $2 \mu \mathrm{L}$ of the processed RNA was used as template, and RT-qPCR was performed. We employed the One Step SYBR PrimeScript RT-PCR Kit II (TaKaRa Bio) and the Chromo 4 real time PCR system (BioRad, Berkeley, CA) for detection. RT-qPCR was done following the manufacturer's instructions with HBV primers specific to pgRNA spanning nucleotides 2429-2636 (forward: 5'-CTCAATCTCGGGAATCTCAATGT-3', reverse 5'-TGGATAAAACCTAGCAGGCATAAT-3') (13). Briefly, for the reverse transcription reaction, the samples were incubated for $5 \mathrm{~min}$ at $42^{\circ} \mathrm{C}$ and then at $95^{\circ} \mathrm{C}$ for $10 \mathrm{~s}$. For amplification by PCR, cDNA was subjected to 40 cycles of 


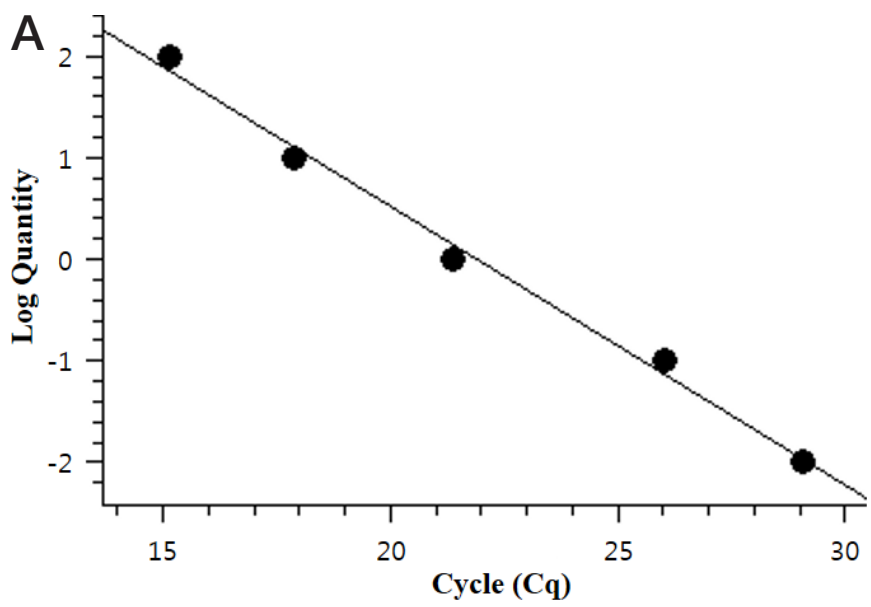

C

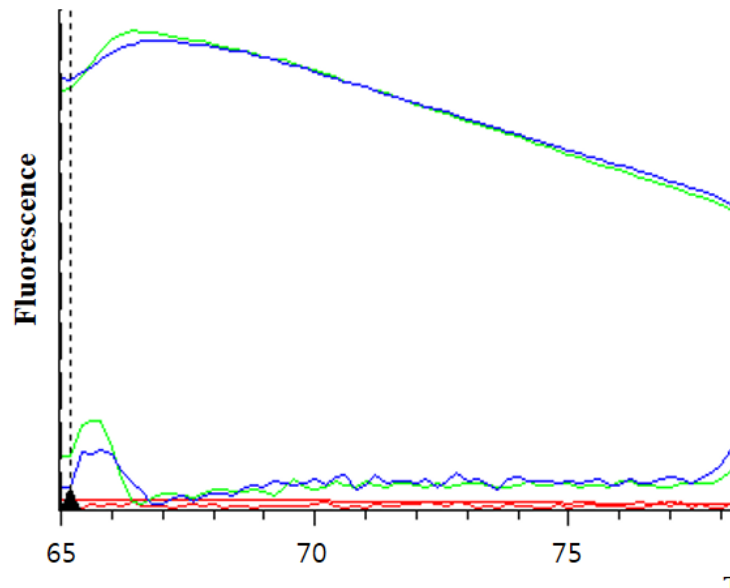

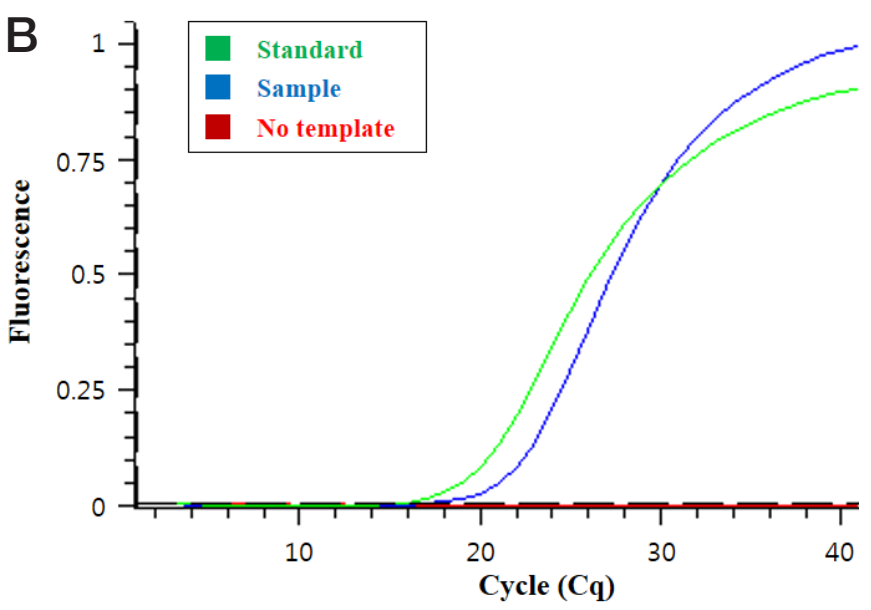

\section{Temperature $\left({ }^{\circ} \mathrm{C}\right)$}

Figure 1. Detection of encapsidated hepatitis B virus (HBV) pregenomic RNA (pgRNA) in 96-well plates by nucleocapsid-captured quantitative RT-PCR ( NCC RT-qPCR). (A) Standard curve analysis with 10-fold serial diluted viral pgRNAs in nucleocapsids by polyethylene glycol (PEG) (Standard). Each data point corresponds to an input pgRNA amount ranging from 100 to $0.01 \mathrm{ng}$. The $\mathrm{x}$-axis and $\mathrm{y}$-axis depict PCR quantitation cycle $(\mathrm{Cq})$ and Log pgRNA quantity, respectively. (B) Amplification curve analysis with representative samples and standard pgRNA. The $\mathrm{x}$-axis and $\mathrm{y}$-axis indicate cycle number for amplification and fluorescence intensity, respectively. Cq is the cycle number at which fluorescence generated within a reaction crosses the threshold line. One representative sample (blue) in the absence of compounds was depicted. Standard pgRNA (green) was employed as positive control. No template $\left(\mathrm{H}_{2} \mathrm{O}\right.$ ) was used as a negative control (red). (C) Melting curve analysis. The $x$-axis and $y$-axis depict temperature $\left({ }^{\circ} \mathrm{C}\right)$ and fluorescence intensity, respectively. Single representative samples in the absence of compounds (blue), with standard pgRNA (green), or with no template (red) are indicated. Experiments were performed in triplicate.

denaturation for $10 \mathrm{~s}$ at $95^{\circ} \mathrm{C}$, annealing for 10 s at $62^{\circ} \mathrm{C}$, and extension for 10 s at $72^{\circ} \mathrm{C}$.

Native agarose gel

analysis of nucleocapsids

Native agarose gel analysis of viral nucleocapsids (core particles) was performed as described previously (7,9-11). HepG2 cells were transfected with a 1.3-mer HBV wild type (WT)-replicon by PEI (polyethyleneimine, MW 25,000; Polysciences, Inc., PA). Next, the transfected cells were treated with DVR-45 compound twice for 2 days. Three days post-transfection, the cells were harvested and lysed with NP40 lysis buffer. Nucleocapsids were precipitated from supernatants of transfected cells by PEG as previously described (7). Capsids were pelleted by centrifugation at $13,000 \times g$ for $15 \mathrm{~min}$, resus- pended in NP40 lysis buffer, and mixed with $6 \times$ loading buffer [ $50 \%$ glycerol, $0.1 \%$ bromophenol blue (BPB)]. The sample was subjected to $1.2 \%$ agarose gel electrophoresis, followed by protein transfer to a PVDF membrane (Immobilon-P; Merck Millipore, Darmstadt, Germany) through capillary action in $10 \times$ SSC $(1.5 \mathrm{M} \mathrm{NaCl}$, $0.15 \mathrm{M}$ sodium citrate) buffer. Nucleocapsids were detected by rabbit anti-core antibody (1:5000) (DAKO), followed by detection with anti-rabbit horseradish peroxidase-linked antibody (Amersham/ GE Healthcare, Little Chalfont, United Kingdom). To quantify encapsidated RNA, capsid-associated RNA was detected by an HBV core-specific riboprobe (5) from the same membrane following UV-crosslinking. The encapsidation ratio (\%) was calculated by the following equation:
(pgRNA - background) / (nucleocapsids - background) $\times 100$. The DMSO control encapsidation ratio was set to $100 \%$.

\section{Results and discussion}

We devised a novel method using 96-well plates to conveniently determine HBV pgRNA encapsidation by utilizing capture plates to immobilize nucleocapsids, followed by the detection of viral pgRNA via RT-qPCR. The cell lysates prepared from HBV-replicating cells were incubated in 96-well capture plates coated with anti-HBc antibody, followed by proteinase $\mathrm{K}$ and DNase I treatment to degrade nucleocapsids and viral DNA, respectively. This step ensures that nucleocapsids are captured by HBc-specific antibodies, proteinase treatment digests the nucleocapsids and releases pgRNA, and 
the DNase treatment prevents the detection of already replicated HBV DNA genomes. The liberated viral RNA was analyzed by RT-qPCR using HBV pgRNA-specific primers and SYBR green.

To establish a novel HBV encapsidation assay with increased sample throughput, we validated our RT-qPCR protocol in 96-well plates using pgRNA (standard) prepared by PEG precipitation of nucleocapsids as reported previously (7). HBV-replicating cells plated in 12-well plates are lysed in NP40 buffer, and clarified cell lysates are treated with DNase I and micrococcal nuclease to remove intracellular DNA and RNA that is not encapsidated. Afterwards, nucleocapsids are precipitated by PEG, and pgRNA is extracted by phenol-chloroform. This pgRNA was used as a standard and analyzed by RT-qPCR using pgRNA-specific primers (13). The pgRNA (standard) was 10-fold serially diluted starting from $100 \mathrm{ng}$ and validated by a 5-point dose-response curve analysis. As shown in Figure $1 \mathrm{~A}$, the results suggest that RT-qPCR conditions were acceptable, as demonstrated by a PCR efficiency of $89.7 \%$, with a coefficient of correlation $\left(R^{2}\right)$ of 0.994 and a slope of -3.595 .

To investigate whether pgRNA prepared in 96-well plates with our new protocol (sample) could be detected by RT-qPCR, we compared it to conventionally PEG-prepared pgRNA (standard) that was used as a positive control and to the negative control (no template) by amplification curve and melting curve analysis (Figure 1B). The amplification curve analysis demonstrated that the pgRNA purified from HepG2.2.15 cells in 96-well plates by our new method was detectable and that the amount of pgRNA sample was comparable to $1 \mathrm{ng}$ of the PEG-prepared pgRNA. In contrast, the no template control did not result in any amplified PCR product (Figure 1B). Importantly, the melting curve analysis demonstrated that the PCR product is HBV pgRNAspecific and that primer dimers were not formed, whereas the no template control did not produce amplicons, demonstrating the HBV-specificity of our assay (Figure 1C). To our knowledge this is the first time that encapsidated pgRNA from HBV replicating cells has been detected in a 96-well plate format.

In order to further validate our assay, we evaluated the effect of DVR-45, a drug that was recently reported to prevent HBV assembly (8). HepG2.2.15 cells plated in a 96-well plate were treated with serially diluted
DVR-45, nucleocapsids were prepared 9 days post-treatment, and RT-qPCR was carried out as described above. By doseresponse curve analysis, we determined the effective concentration $\left(\mathrm{EC}_{50}\right)$ to inhibit $50 \%$ HBV pgRNA encapsidation, and the cytotoxic concentration $\left(\mathrm{CC}_{50}\right)$ was determined in parallel using a resazurin-based assay (Figure 2A). Importantly, our new encapsidation assay demonstrated that DVR-45 treatment reduces pgRNA levels in nucleocapsids in a dose-dependent manner without showing any cytotoxicity over the dose range employed $\left(\mathrm{EC}_{50} 2.57 \mu \mathrm{M}\right.$ and $\mathrm{CC}_{50}>20 \mu \mathrm{M}$ in HepG2.2.15 cells).

In order to corroborate the inhibitory effect of DVR-45 revealed by our novel PCR-based encapsidation detection assay, we compared it with native capsid gel electrophoresis, which is still one of the most frequently applied techniques for monitoring encapsidation inhibition. Briefly, cells were transfected with 1.3-mer WT HBV DNA (6), and starting from 1 day post-transfection, cells were treated daily with serially diluted HBV encapsidation inhibitor DVR-45 and RT inhibitor lamivudine (LMV). At day 3 post-transfection, cells were harvested, capsids were precipitated, and the encapsidated pgRNA was examined as detailed above. As shown in Figure 2B, increasing the concentration of DVR-45 did not affect HBV capsid particle formation (upper panel), whereas encapsidated pgRNA decreased in a dose-dependent manner (lower panel). This result indicates that DVR-45 inhibits the viral pgRNA packaging at an $\mathrm{EC}_{50}$ value of approximately $0.5 \mu \mathrm{M}$, which corroborates our data and is further substantiated by data recently reported by Campagna et al. employing Southern blotting analysis ( $\mathrm{EC}_{50}$ $0.46 \pm 0.38 \mu \mathrm{M}$ and $\left.\mathrm{CC}_{50}>50 \mu \mathrm{M}\right)(8)$. It is interesting to note that the amount of encapsidated pgRNA seems to be increased in the presence of LMV, which might be a consequence of the lack of viral RNA degradation by RNase H activity as a consequence of RT inhibition by LMV (Figure 2B, lower panel).

We then investigated the antiviral effect of another pgRNA encapsidation inhibitor, AT-130, a phenylpropenamide derivative that was reported to interfere with encapsidation $\left(\mathrm{EC}_{50} 2.5 \mu \mathrm{M}\right.$ and $\mathrm{CC}_{50}>250 \mu \mathrm{M}$ in HepG2 cells infected by recombinant HBV-baculovirus) (14). As expected, AT-130 also inhibited encapsidated pgRNA levels in a non-toxic and dose-dependent manner in our NCC RT-qPCR assay $\left(\mathrm{EC}_{50} 2.06 \mu \mathrm{M}\right.$ and $\mathrm{CC}_{50}>$ $20 \mu \mathrm{M}$ in HepG2.2.15 cells) (Figure 2C). As a DNA

Bacterial Colonies
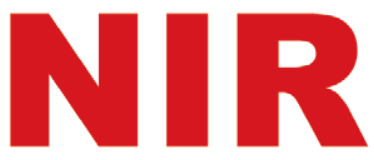

Proteins

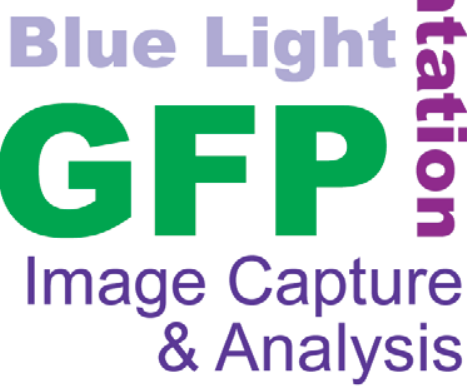

Colorimetric
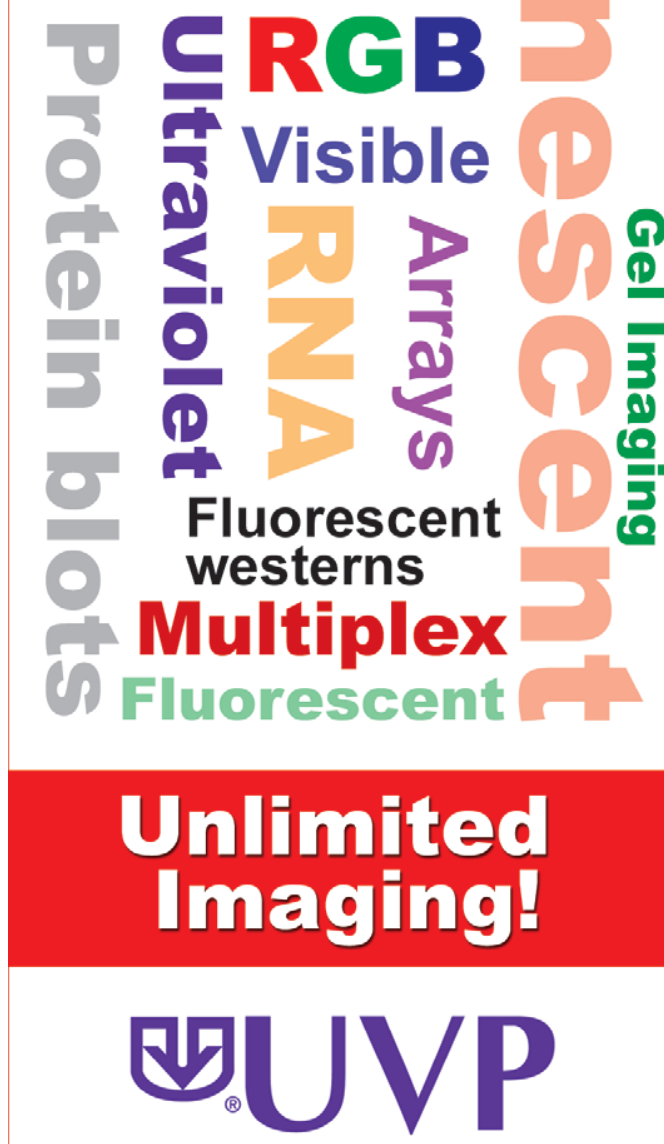

An Analytik Jena Company

UVP, LLC USA: (800) 452-6788 | (909) 946-3197 Cambridge UK: +44(0)1223 420022 


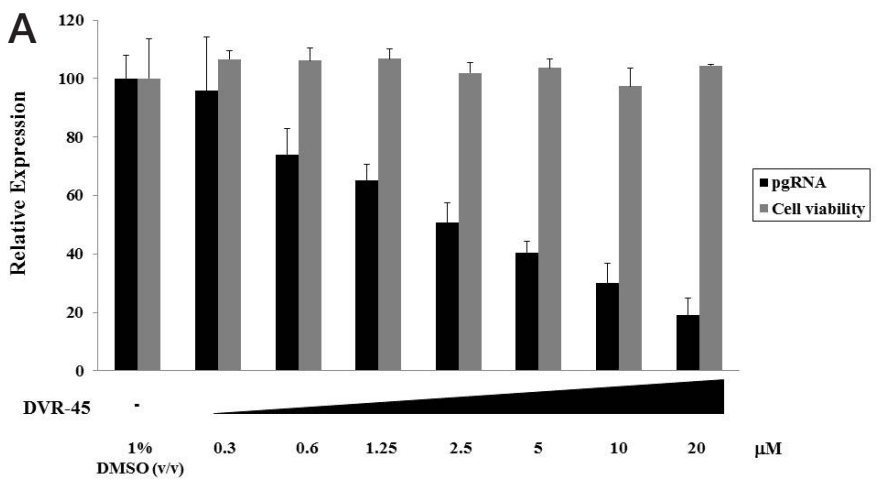

B
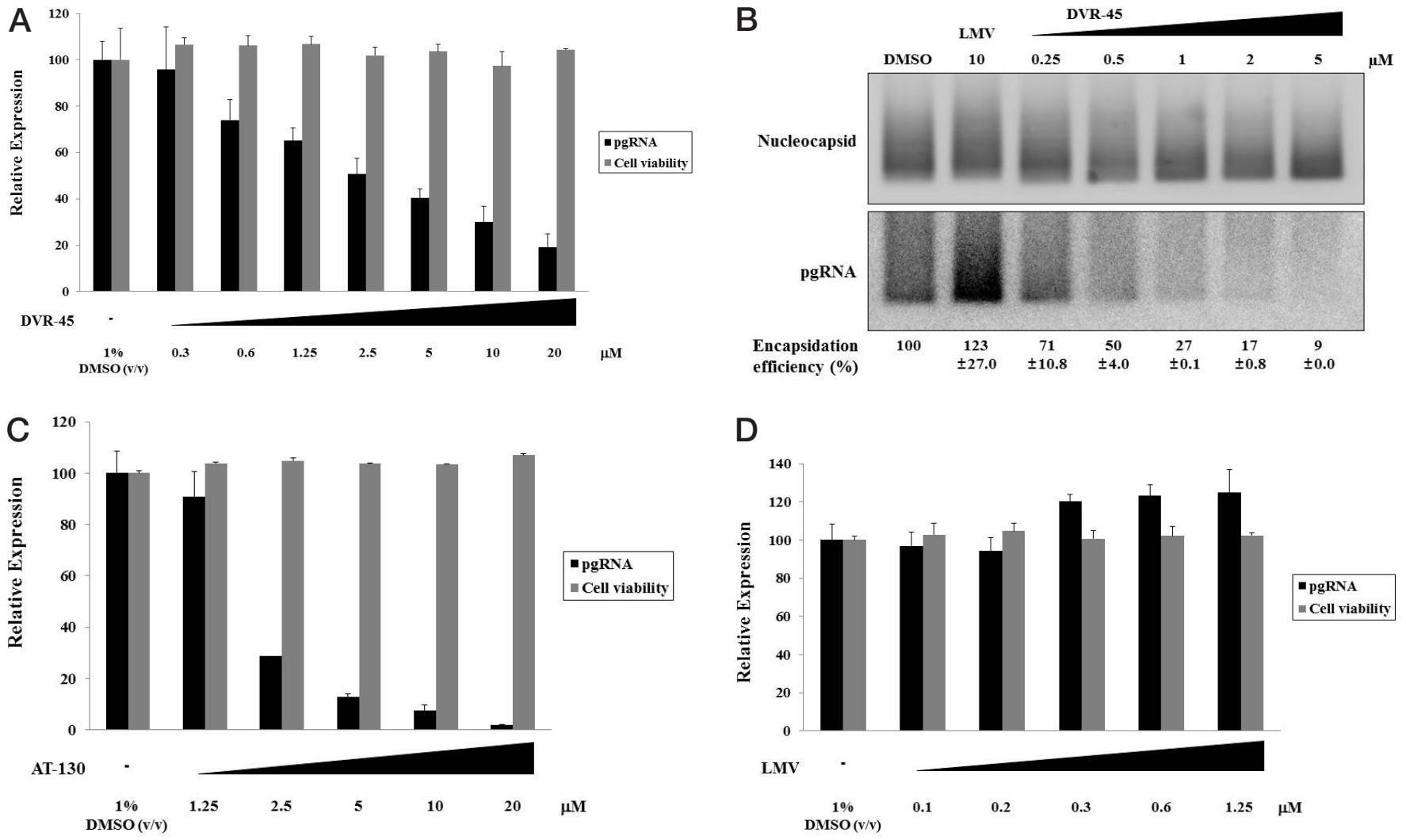

Figure 2. Validation of nucleocapsid-captured (NCC) quantitative RT-PCR (RT-qPCR) using hepatitis B virus (HBV) encapsidation and reverse transcriptase (RT) inhibitors. (A) Validation of NCC RT-qPCR by dose-response curve analysis using encapsidation and replication inhibitors. HepG2.2.15 cells were treated with inhibitors and analyzed by RT-qPCR 9 days post-treatment as described in the "Materials and methods" section. Relative HBV RNA expression levels were quantified in triplicate (black bars) and normalized to the DMSO (v/v) control, which was set to $100 \%$. In parallel, cytotoxicity was determined by a resazurin-based assay (gray bars) Evaluation of the HBV encapsidation inhibitor DVR-45 at the indicated concentrations (20 to $0.3 \mu \mathrm{M}$ ). (B) Evaluation of pregenomic RNA (pgRNA) encapsidation and RT inhibitors by native agarose gel electrophoresis. Viral nucleocapsids isolated as described in the "Materials and methods" section from HBV replicating cells were treated with encapsidation or replication inhibitors, DVR-45 and lamivudine (LMV), respectively, and analyzed by native agarose gel electrophoresis. After protein transfer to a PVDF membrane, viral core protein was detected by an HBc-specific antibody (top), and viral RNA was detected by hybridization to an HBV-specific riboprobe (bottom). Encapsidation ratio efficiency was calculated as described in the Materials and methods section. (C) Evaluation of HBV encapsidation inhibitor AT130 and (D) replication inhibitor LMV at indicated concentrations using NCC RT-qPCR as described in (A). Representative experiments are shown.

negative control incapable of interfering with HBV capsid assembly, we employed LMV, an nucleoside analog that has been used for nearly 2 decades for clinical treatment of $\mathrm{CHB}$ with a reported $\mathrm{EC}_{50}$ range of 0.05-0.3 $\mu \mathrm{M}$ in HepG2.2.15 cells $(15,16)$. LMV was tested by 5-point dose-response curve analysis within the range of physiologically effective concentrations. As expected, no effect on encapsidation of pgRNA into HBV nucleocapsids was detectable (Figure 2D), substantiating that only compounds interfering with capsid assembly are active in this NCC RT-qPCR assay. Furthermore, in the presence of LMV, encapsidated pgRNA levels seem to be increased, corroborating data obtained by our native capsid gel electrophoresis analysis shown in Figure 2B.

As demonstrated here, we successfully developed a novel HBV encapsidation assay by using capture plates, usually used for ELISA, to immobilize HBV nucleocapsids in order to detect encapsi- dated pgRNA via RT-qPCR. We produced nucleocapsid capture plates by immobilizing anti-HBc antibody in 96-well plates, which were then used to capture HBV pgRNA-containing nucleocapsids that were liberated by proteinase K treatment. Subsequently, pgRNA-specific RT-qPCR was performed in a 96-well plate. We validated this novel NCC RT-qPCR assay with encapsidated pgRNA isolated by PEG and with recently reported encapsidation inhibitors (DVR-45 and AT-130) in stably HBV-replicating HepG2.2.15 cells. In addition, we corroborated our NCC RT-qPCR assay data with results obtained by native agarose gel electrophoresis analysis. One hallmark of the NCC RT-qPCR assay is its simplicity of pgRNA sample processing and detection by PCR, overcoming the limitations of encapsidation analysis by RNase protection or native agarose gel assays, which are unable to handle a large number of samples in parallel. Furthermore, the NCC RT-qPCR assay supports the quantitative analysis of encapsidation inhibition by dose-response curve analysis to precisely evaluate antiviral efficacy. With the newly discovered role of sodium taurocholate cotransporting polypeptide (NTCP) as an entry receptor for $\mathrm{HBV}$, this infectious cell culture system will open new avenues for drug discovery (17). Using this system for target-free screening campaigns, target identification and hit characterization studies employing sophisticated secondary assays are required. Our pgRNA encapsidation assay requires significantly fewer experimental steps than other encapsidation assays, (e.g., no gel electrophoresis, membrane transfer, preparation of riboprobes, hybridization, etc.), consequently reducing assay variation. Accordingly, this assay is convenient for triaging inhibitors identified by screens using HBV-replicating cells. In addition, our assay is potentially suitable for highthroughput screening (HTS) with further 
development. In order to develop an assay that suits HTS requirements, assay robustness and reproducibility have to be determined by evaluating inter- and intraplate variation. Furthermore, the assay has to be adapted to automation (e.g., cell plating, liquid handling, read-out, analysis, etc.), enabling the handling of a high number of assay plates.

Concerning potential drugs interfering with encapsidation of pgRNA into HBV capsids, if these are combined with RT inhibitors, encapsidation inhibitors could add value to existing therapies by reducing the emergence of viral drug resistance and potentially might cure $\mathrm{CHB}$ by preventing the re-import of HBV genomes into the host's nuclei, thereby abolishing the replenishment of viral covalently closed circular DNA (cccDNA) pools.

In summary, we developed a HBV pgRNA encapsidation assay without using radioisotopes that is safer and cheaper than previously described methods. Additionally, our NCC RT-qPCR assay supports the rapid and quantitative analysis of multiple samples in parallel and opens avenues for identifying and characterizing novel HBV inhibitors.

\section{Author contributions}

D.K.R., W.S.R., and M.P.W. designed experiments. D.K.R. and Y.A. performed the experiments. D.K.R. and M.P.W. analyzed data, interpreted results, and wrote the manuscript. All authors read and approved the manuscript.

\section{Acknowledgments}

AT-130 was kindly provided by William E. Delaney at Gilead Sciences. The authors are grateful to Sang-Hyun Park, Peter Sommer, and HeeYoung Kim for technical support and advice. This work was supported by the National Research foundation of Korea (NRF-2014K1A4A7A01074644) grant funded by the Korea government (MSIP), Gyeonggi-do and KISTI.

\section{Competing interests}

The authors declare no competing interests.

\section{References}

1. Zoulim, F. 2006. Antiviral therapy of chronic hepatitis B. Antiviral Res. 71:206-215.

2. Zoulim, F. and S. Locarnini. 2009. Hepatitis B virus resistance to nucleos(t)ide analogues. Gastroenterology. 137:1593-1608.e1-2.
3. Seeger, C. and W.S. Mason. 2000. Hepatitis B virus biology. Microbiology and molecular biology reviews. Microbiol. Mol. Biol. Rev. 64:51-68.

4. Ryu, D.K., B.Y. Ahn, and W.S. Ryu. 2010. Proximity between the cap and 5' epsilon stem-loop structure is critical for the suppression of pgRNA translation by the hepatitis B viral polymerase. Virology 406:56-64.

5. Ryu, D.K., S. Kim, and W.S. Ryu. 2008. Hepatitis B virus polymerase suppresses translation of pregenomic RNA via a mechanism involving its interaction with 5' stem-loop structure. Virology 373:112-123.

6. Cha, M.Y., D.K. Ryu, H.S. Jung, H.E. Chang, and W.S. Ryu. 2009. Stimulation of hepatitis B virus genome replication by $\mathrm{HBx}$ is linked to both nuclear and cytoplasmic HBx expression. J. Gen. Virol. 90:978-986.

7. Jeong, J.K., G.S. Yoon, and W.S. Ryu. 2000. Evidence that the 5 -end cap structure is essential for encapsidation of hepatitis B virus pregenomic RNA. J. Virol. 74:5502-5508.

8. Campagna, M.R., F. Liu, R. Mao, C. Mills, D. Cai, F. Guo, X. Zhao, H. Ye, et al. 2013. Sulfamoylbenzamide derivatives inhibit the assembly of hepatitis B virus nucleocapsids. J. Virol. 87:6931-6942.

9. Melegari, M., S.K. Wolf, and R.J. Schneider. 2005. Hepatitis B virus DNA replication is coordinated by core protein serine phosphorylation and HBx expression. J. Virol. 79:9810-9820.

10. Hu, J., D. Flores, D. Toft, X. Wang, and D. Nguyen. 2004. Requirement of heat shock protein 90 for human hepatitis B virus reverse transcriptase function. J. Virol. 78:13122-13131.

11. Yu, M. and J. Summers. 1994. Multiple functions of capsid protein phosphorylation in duck hepatitis B virus replication. J. Virol. 68:4341-4348.

12. Chomczynski, P. and N. Sacchi. 1987. Single-step method of RNA isolation by acid guanidinium thiocyanate-phenol-chloroform extraction. Anal. Biochem. 162:156-159.

13. Xu, W.S., K.K. Zhao, X.H. Miao, W. Ni, X. Cai, R.Q. Zhang, and J.X. Wang. 2010. Effect of oxymatrine on the replication cycle of hepatitis B virus in vitro. World journal of gastroenterology. World J Gastroenterol. 16:2028-2037.

14. Feld, J.J., D. Colledge, V. Sozzi, R. Edwards, M. Littlejohn, and S.A. Locarnini. 2007. The phenylpropenamide derivative AT-130 blocks HBV replication at the level of viral RNA packaging. Antiviral Res. 76:168-177.

15. Deres, K., C.H. Schroder, A. Paessens, S. Goldmann, H.J. Hacker, O. Weber, T. Kramer, U. Niewohner, et al. 2003. Inhibition of hepatitis B virus replication by drug-induced depletion of nucleocapsids. Science 299:893-896.

16. Ying, C., Y. Li, C.H. Leung, M.D. Robek, and Y.C. Cheng. 2007. Unique antiviral mechanism discovered in anti-hepatitis B virus research with a natural product analogue. Proc. Natl. Acad. Sci. USA 104:8526-8531.

17. Yan, H., G. Zhong, G. Xu, W. He, Z. Jing, Z. Gao, Y. Huang, Y. Qi, et al. 2012. Sodium taurocholate cotransporting polypeptide is a functional receptor for human hepatitis B and D virus. Elife 1:e00049.

Received 02 July 2015; accepted 26 August 2015

Address correspondence to Marc P. Windisch, Hepatitis Research Laboratory, Institut Pasteur Korea, 696 Sampyung-dong, Bundang-gu, Seongnam-si, Gyeonggi-do, South-Korea 463-400. E-mail: marc. windisch@ip-korea.org

To purchase reprints of this article, contact: biotechniques@fosterprinting.com

\section{Non-invasive detection of apoptosis in vivo}

\section{CAS-MAP"' In Vivo Apoptosis Detection}

» Enables chronic, longitudinal in vivo studies

» Unbound probe is rapidly cleared from circulation

» Species independent and works with various cell and tissue types

» Suitable for animal imaging, fluorescent microscopy, plate reader and flow cytometry

» Doesn't label artifacts from ex vivo sample processing giving a true representation of in vivo apoptosis.

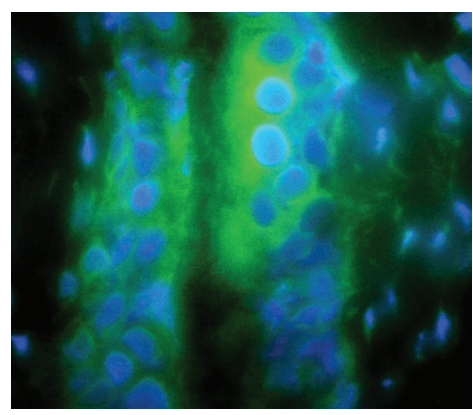

CAS-MAP'M Pan Capase Probe was administered to young rats via IV injection. After 30 minutes the urinary bladder was excised, counterstained with DAPI and anlyzed by fluorescence microscopy. Data courtesy of Dr Andreja Erman, Institute of Cell Biology, Slovenia

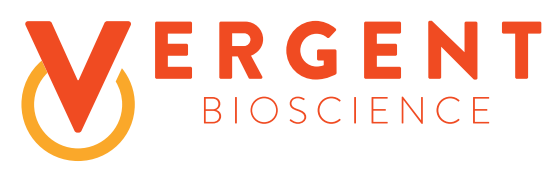

vergentbio.com/invivo $15 \%$ off using code 02-0915 